\title{
Perceived Recovery Time from Common Cold as a Possible Indicator of Physical Resilience
}

\author{
Yoonki Kim ${ }^{1}$, Chang Won Won ${ }^{2,3}$, Sunyoung Kim ${ }^{3}$, ByungSung Kim ${ }^{3}$, Miji Kim ${ }^{4}$ Eunjin Jeong ${ }^{3}$, Jisoo Yang ${ }^{3}$, Hyona Lee ${ }^{1}$ \\ ${ }^{1}$ Department of Family Medicine, Kyung Hee University Medical Center, Seoul, Korea \\ ${ }^{2}$ Elderly Frailty Research Center, Department of Family Medicine, Kyung Hee University College of Medicine, Seoul, Korea \\ ${ }^{3}$ Department of Family Medicine, Kyung Hee University College of Medicine, Seoul, Korea \\ ${ }^{4}$ Department of Biomedical Science and Technology, East-West Medical Research Institute, Kyung Hee University College of Medicine, Seoul, Korea
}

\author{
Corresponding Author: \\ Chang Won Won, $\mathrm{MD}, \mathrm{PhD}$ \\ Elderly Frailty Research Center, \\ Department of Family Medicine, \\ Kyung Hee University College of \\ Medicine, Kyungheedaero 23, \\ Dongdaemun-gu, Seoul 02447, Korea \\ Tel: +82-2-958-8700 \\ E-mail: chunwon62@naver.com \\ ORCID:
}

https://orcid.org/0000-0002-6429-4461

Received: May 26, 2021

Revised: June 28, 2021

Accepted: June 29, 2021

\begin{abstract}
Background: Resilience refers to the ability to recover function after encounter with stressors. While psychological resilience refers to the ability to cope with psychological stress, physical resilience refers to functional restoration after biomedical challenges. However, there is no gold standard to assess physical resilience. Accordingly, we explored whether the perceived recovery time from common cold could be used to represent physical resilience. Methods: We analyzed data of individuals aged 72-86 years who had participated in the Korean Frailty and Aging Cohort Study in 2019. Among the 1,455 survey participants, 594 with asthma and chronic obstructive pulmonary disease and missing data were excluded. The remaining 861 participants were divided into three groups according to the number of days required for recovery from common cold (Group 1, 1-4 days; Group 2, 5-7 days; and Group 3, $\geq 8$ days). The relationship between recovery time and psychological resilience scale (Brief Resilience Scale [BRS]) score, physical frailty (Fried's physical frailty phenotype and the Korean Frailty Index for Primary Care [KFI-PC]), and frailty outcome was investigated. Results: Group 3 comprised individuals more likely to be women, sleep less, be less physically less active, fall more often, and have a low EuroOol visual analogue scale score. BRS scores differed significantly among the three groups (Group 1, 13.29; Group 2, 14.32; Group 3, 15.22; $p<0.001)$. In multivariate analysis, post-hoc analysis with the Bonferroni method revealed significant differences in BRS between Groups 1-2 and Groups 1-3. However, the KFI-PC and number of falls did not differ significantly. Conclusions: Longer days of recovery from cold were associated with worse BRS scores. However, neither frailty nor the number of falls was related.
\end{abstract}

Key Words: Common cold, Frailty, Psychological resilience

\section{INTRODUCTION}

Frailty is defined as a significant decline in the functional reserve of multiple organ systems and the resultant extreme vulnerability of an individual to endogenous and exogenous stressors (such as infection, injury, surgery, or some medicines), leading to a higher risk of accelerated functional decline and adverse health-related outcomes. ${ }^{1)}$ The adverse health-related outcomes caused by frailty in- clude falls, delirium, immobility, or disability, and, consequently, hospitalizations, institutionalization, or mortality. ${ }^{1}$

Over the past decade, resilience has increasingly become a focus of research in the behavioral and medical sciences. Frail older adults have a preexisting vulnerability to stressors, often resulting in poor outcomes. However, some people recover rapidly from stressors without such outcomes. These individuals are categorized as resilient. Resilience has been defined in various ways, in- 
cluding the ability to rapidly recover from various stressors. ${ }^{2)}$ The factors that promote resilience, including physical health, a sense of self, social belonging, self-belief, and altruism, overlap with those that reduce frailty. ${ }^{3)}$ Therefore, an understanding of resilience may help in the study of frailty. Furthermore, a deeper understanding of why some individuals maintain or regain function following stressors, while others do not, may help identify protective factors and strategies to promote lasting health. ${ }^{4)}$

There are two types of resilience. The first is "psychological resilience," which refers to a person's ability to adapt well in the face of adversity, trauma, tragedy, threats, or significant sources of stress. The second is "physical resilience," which focuses on the maintenance or recovery of functions after biomedical or pathological challenges. Physical resilience is presumed to reflect adaptive physiological responses at the level of organs, cells, and molecules of the musculoskeletal, neurological, and immunological systems that support homeostasis under changing conditions. ${ }^{5)}$

Psychological resilience is measured with well-organized standard assessments such as the Brief Resilience Scale (BRS). ${ }^{6}$ However, there is no gold standard test for measuring physical resilience.

We hypothesized that the time needed to recover from a common cold could be an indicator of physical resilience, as the immunologic response is one component of physical resilience. ${ }^{5)} \mathrm{A}$ previous study showed that self-reported health was related to the duration of common cold. ${ }^{7)}$ Therefore, we investigated whether the day(s) required for recovery from common cold could represent physical resilience by comparing this number with BRS scores, physical frailty, and health variables to ensure validity.

\section{MATERIALS AND METHODS}

\section{Participants}

The Korean Frailty and Aging Cohort Study (KFACS) is a multicenter longitudinal study with a baseline survey conducted from 2016 to 2017 in Korea with follow-ups every 2 years. The inclusion criteria of the KFACS were age 70-84 years, currently living in the community, no plans to move out in the next 2 years, and no problems with communication and no prior dementia diagnosis. ${ }^{8)}$ In 2018, 1,455 participants aged 72-86 years who were included in the study in 2017 were asked to complete a questionnaire on the time required to recover from a common cold. Patients with asthma and chronic obstructive pulmonary disease $(n=145)$ were excluded from the analysis because they may not be able to differentiate common cold from their disease. ${ }^{9)}$ Participants who answered, "have never caught a cold" $(\mathrm{n}=320)$ were also excluded. Finally, those who did not answer the entire questionnaire were also excluded (Fig. 1).

\section{Measures}

\section{Perceived days to recover from common cold}

We asked the following question: "If you have a common cold, how many days does it usually take for recovery?" (Based on the start date of the cold).

\section{Brief Resilience Scale}

We used the Korean version of the BRS to assess psychological resilience. ${ }^{10)}$ The six items of the BRS are (1) I tend to bounce back quickly after hard times, (2) I have a hard time making it through stressful events (R), (3) It does not take me long to recover from a stressful event, (4) It is hard for me to bounce back when something bad happens (R), (5) I usually come through difficult times with little trouble, and (6) I tend to take a long time to get over setbacks in my life (R). "R" refers to the reverse coding applied to items 2 , 4, and 6 . The following instructions were used to administer the scale: "Please indicate the extent to which you agree with each of the following statements using the following scale: $1=$ strongly agree, 2 = agree, 3 = neutral, $4=$ disagree, and 5 = strongly disagree." We summed these scores, with higher scores indicating worse psychological resilience. ${ }^{2)}$

\section{Frailty and frailty outcome}

Frailty: We defined frailty using Fried's physical frailty phenotype $^{11)}$ and the Korean Frailty Index for Primary Care (KFI-PC). The KFI-PC comprehensively evaluates many aspects of physical, social, and emotional health. ${ }^{12)}$

Frailty outcomes: The frailty outcomes included the number of hospitalizations and falls over the past year, along with instrumental activities of daily living (IADL) disability. IADL disability was

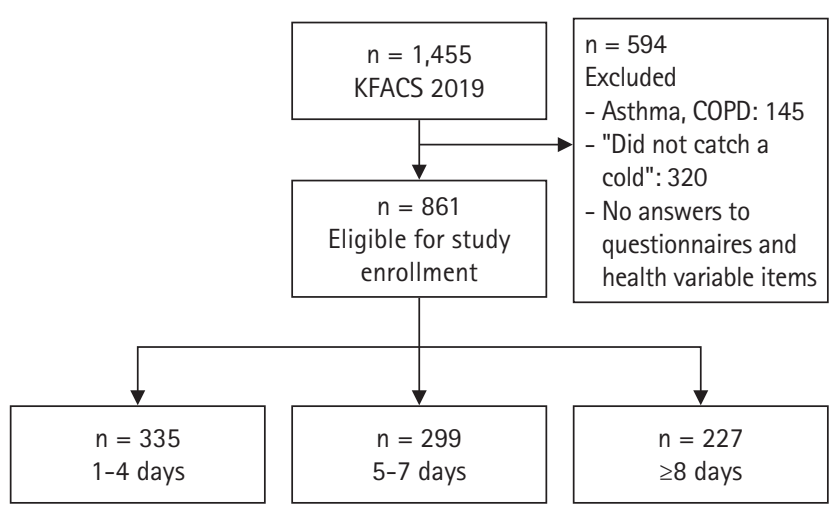

Fig. 1. Study flowchart. KFACS, Korean Frailty and Aging Cohort Study; COPD, chronic obstruction pulmonary disease. 
defined as the need for assistance in one or more of the 10 items. The answer "I do not know" was treated as a missing value.

\section{Ethical Approval}

The study protocol was approved by the Institutional Review Board of Kyung Hee University Hospital (No. 2021-03-060) and complied with the ethical rules for human experimentation in the Declaration of Helsinki. Informed consent was obtained from all participants or their proxy.

\section{Statistical Analysis}

We used the analysis of variance and chi-square test for continuous and categorical variables, respectively. Analysis of covariance (ANCOVA) was used to control for confounding variables.

\section{RESULTS}

The participants were divided into three groups according to the numbers of perceived days required for recovery from common cold (Group 1, 1-4 days; Group 2, 5-7 days; Group 3, $\geq 8$ days) (Table 1). The mean ages of the three groups did not differ significantly, and the mean age of all subjects was approximately 78 years. Participants in Group 3 ( $\geq 8$ days for recovery) were more likely to be women $(60.8 \%, p=0.004)$, sleep less, less physically active, fall more, and have a low EuroQol visual analogue scale (EQ-VAS) score. The BRS scores differed significantly among the three groups (Group 1, 13.29; Group 2, 14.32; Group 3, 15.22; $\mathrm{p}<0.001$ ). The KFI-PC was higher in Group 3 than in other groups (Group 1, 0.15; Group 2, 0.16; Group 3, 0.17; p = 0.034).

Tables 2-4 show the results of ANCOVA. Model 1 was adjusted for sex, Model 2 was further adjusted for sleep time, Model 3 was additionally adjusted for IPAQ and Model 4 was additionally adjusted for EQ-VAS scores. Table 2 shows the differences in BRS scores among the three groups. Even after adjusting for the aforementioned factors, the difference between the three groups was significant in Model 4 (Group 1, 13.55; Group 2, 14.32; Group 3, 14.84; $\mathrm{p}<0.001)$. Table 3 presents the relationships with the number of falls and shows no significant results. Table 4 shows the results for KFI-PC, in which we observed no significant differences.

The Bonferroni method was used for post-hoc analysis in Tables 2-4. In Table 2, which was related to the BRS score, the differences between Groups 1 and 2 in Models 1, 2, and 3 were significant. The difference between Groups 1 and 3 was also significant after adjusting for all confounding factors. In Table 3, which represented the number of falls, we observed a significant difference between the groups only for Model 1. We observed no significant difference in KFI-PC.

Table 1. Participant characteristics

\begin{tabular}{|c|c|c|c|c|}
\hline \multirow{2}{*}{ Characteristic } & \multicolumn{3}{|c|}{ Perceived days required to recover from common cold } & \multirow{2}{*}{ p-value } \\
\hline & $1-4(n=335)$ & $5-7(n=299)$ & $\geq 8(n=227)$ & \\
\hline Age (y) & $77.9 \pm 4.0$ & $77.9 \pm 3.7$ & $77.5 \pm 3.5$ & 0.469 \\
\hline Sex, female & $158(47.2)$ & $146(48.8)$ & $138(60.8)$ & 0.004 \\
\hline Income per month $(<2,000,000$ Korean won $)$ & $217(64.8)$ & $171(57.2)$ & $148(65.2)$ & 0.082 \\
\hline Education $(y)$ & $9.7 \pm 4.8$ & $9.8 \pm 6.9$ & $8.9 \pm 7.4$ & 0.190 \\
\hline EQ-VAS score & $77.1 \pm 15.9$ & $75.1 \pm 16.6$ & $72.6 \pm 17.9$ & 0.008 \\
\hline Sleep at night $(\mathrm{hr})$ & $6.4 \pm 1.5$ & $6.3 \pm 1.4$ & $6.0 \pm 1.4$ & 0.004 \\
\hline MNA, malnutrition risk $($ score $<12)$ & $52(15.5)$ & $52(17.4)$ & $43(18.9)$ & 0.562 \\
\hline $\operatorname{IPAQ}(\mathrm{cal} / \mathrm{wk})$ & $3,648.3 \pm 3,959.6$ & $3,670.0 \pm 3,977.6$ & $2,906.9 \pm 2,737.7$ & 0.031 \\
\hline Number of chronic diseases & $2.0 \pm 1.4$ & $1.9 \pm 1.4$ & $2.2 \pm 1.4$ & 0.134 \\
\hline Number of medications & $3.9 \pm 2.9$ & $4.1 \pm 3.2$ & $4.3 \pm 3.2$ & 0.215 \\
\hline Number of hospital admissions in the last year & $0.2 \pm 0.6$ & $0.2 \pm 0.5$ & $0.2 \pm 0.4$ & 0.301 \\
\hline Number of falls in the last year & $0.3 \pm 0.7$ & $0.5 \pm 1.9$ & $0.5 \pm 1.1$ & 0.041 \\
\hline IADL disability & $13(3.9)$ & $12(4.0)$ & $11(4.9)$ & 0.841 \\
\hline Brief Resilience Scale score & $13.3 \pm 4.3$ & $14.3 \pm 4.7$ & $15.2 \pm 5.2$ & $<0.001$ \\
\hline Physical frailty & $0(0)$ & $14(4.7)$ & $15(6.6)$ & 0.599 \\
\hline KFI-PC score & $0.15 \pm 0.07$ & $0.16 \pm 0.08$ & $0.17 \pm 0.08$ & 0.034 \\
\hline
\end{tabular}

Values are presented as mean \pm standard deviation or number (\%).

EQ-VAS, EuroQol visual analogue scale; MNA, Mini Nutritional Assessment; IPAQ, International Physical Activity Questionnaire; IADL, instrumental activities of daily living; KFI-PC, Korean Frailty Index for Primary Care. 
Table 2. Brief Resilience Scale scores according to the number of days required to recover from common cold

\begin{tabular}{lcccc}
\hline & \multicolumn{2}{c}{ Perceived days required to recover from common cold } & \\
\cline { 2 - 4 } & $1-4(\mathrm{n}=335)$ & $5-7(\mathrm{n}=299)$ & $28(\mathrm{n}=227)$ & $<0.001$ \\
Model 1 & $13.33 \pm 0.25$ & $14.35 \pm 0.27^{*}$ & $15.13 \pm 0.31^{*}$ & $<0.001$ \\
Model 2 & $13.40 \pm 0.25$ & $14.34 \pm 0.27^{*}$ & $15.04 \pm 0.31^{*}$ & $<0.001$ \\
Model 3 & $13.40 \pm 0.25$ & $14.35 \pm 0.27^{*}$ & $15.03 \pm 0.31^{*}$ & $<0.001$ \\
Model 4 & $13.55 \pm 0.24$ & $14.32 \pm 0.25$ & $14.84 \pm 0.29^{*}$ & \\
\hline
\end{tabular}

Values are presented as mean \pm standard error.

Model 1, adjusted for sex; Model 2, Model 1 + sleep time at night; Model 3, Model 2 + International Physical Activity Questionnaire score; Model 4, Model 3 + EuroQol visual analogue scale score.

Each model was analyzed using an analysis of covariance model and post-hoc Bonferroni correction tests.

* $\mathrm{p}<0.05$, significant difference compared with the "4 days or less" group.

Table 3. Falls in the past year according to the number of days required to recover from common cold

\begin{tabular}{lcccc}
\hline & \multicolumn{2}{c}{ Perceived days required to recover from common cold } & p-value \\
\cline { 2 - 4 } & $1-4(\mathrm{n}=335)$ & $5-7(\mathrm{n}=299)$ & $\geq 8(\mathrm{n}=227)$ & 0.049 \\
Model 1 & $0.28 \pm 0.07$ & $0.53 \pm 0.08^{*}$ & $0.42 \pm 0.09$ & 0.069 \\
Model 2 & $0.29 \pm 0.07$ & $0.53 \pm 0.08$ & $0.43 \pm 0.09$ & 0.069 \\
Model 3 & $0.29 \pm 0.07$ & $0.53 \pm 0.08$ & $0.40 \pm 0.09$ & 0.108 \\
\hline
\end{tabular}

Values are presented as mean \pm standard error.

Model 1, adjusted for sex; Model 2, Model 1 + sleep time at night; Model 3, Model 2 + International Physical Activity Questionnaire score; Model 4, Model 3 + EuroQol visual analogue scale score.

Each model was analyzed using an analysis of covariance model and post-hoc Bonferroni correction tests.

* $\mathrm{p}<0.05$, significant difference compared with the "4 days or less" group.

Table 4. KFI-PC according the number of days required to recover from common cold

\begin{tabular}{lllll}
\hline & \multicolumn{2}{c}{ Perceived days required to recover from common cold } & \multirow{2}{*}{ p-value } \\
\cline { 2 - 4 } & $1-4(\mathrm{n}=335)$ & $5-7(\mathrm{n}=299)$ & $0.167 \pm 0.005$ & 0.187 \\
Model 1 & $0.155 \pm 0.004$ & $0.163 \pm 0.004$ & $0.166 \pm 0.005$ & 0.231 \\
Model 2 & $0.156 \pm 0.004$ & $0.163 \pm 0.004$ & $0.165 \pm 0.005$ & 0.318 \\
Model 3 & $0.156 \pm 0.004$ & $0.163 \pm 0.004$ & $0.161 \pm 0.005$ & 0.777 \\
\hline
\end{tabular}

Values are presented as mean \pm standard error.

KFI-PC, Korean Frailty Index for Primary Care; Model 1, adjusted for sex; Model 2, Model $1+$ sleep time at night; Model 3, Model $2+$ International Physical Activity Questionnaire score; Model 4, Model $3+$ EuroQol visual analogue scale score.

Each model was analyzed using an analysis of covariance model and post-hoc Bonferroni correction tests.

\section{DISCUSSION}

The results of this study showed the association between increased days of recovery from a common cold and lower psychological resilience (BRS score), lower quality of life (EQ-VAS score), and lower physical activity level (IPAQ score). However, we observed no association between recovery days and frailty index and frailty outcomes, such as the number of falls.

It may be not surprising that the number of perceived days required for recovery from a cold was not related to frailty, as frailty refers to a "functionally declined and stressor-vulnerable state," while physical resilience refers to the ability to recover from stressors. Moreover, several factors influence physical resilience, including both emotional and social as well as physical factors. ${ }^{13)}$ Previous studies have shown that an individual's degree of frailty and resilience are not simply opposite concepts. ${ }^{5)}$ Although there is a conceptual overlap between frailty and resilience, frail individuals tend to have a lower resilience and resilience is a continuous spectrum that can change throughout life. In contrast, frailty often evolves near the end of life and manifests in only a small proportion of older adults. ${ }^{5)}$ The lack of association between recovery days from cold and frailty outcomes such as the number of falls 
may be owing to the cross-sectional study design. Thus, longitudinal studies are needed.

Resilience is a dynamic construct that can be ascertained through dynamic stimulation tests. As an alternative approach in an observational study, comparing two measurements-one before and one directly after the stressor-with an outcome is needed. ${ }^{14)}$ We could not present either way in this study, which can be a limitation of this study. Nonetheless, infection is known to be one of the stressors used to evaluate physical resilience, and recovery trajectories after a recent disease (e.g., influenza) is suggested to be one of the best available assessments of resilience. ${ }^{14)}$ In a similar approach, we surveyed the recovery time from common cold, and we believe that it could be an indirect marker for physical resilience, at least with respect to immunity. Dynamic resilience measurements (trajectories, stimulus-response tests) are not yet sufficiently robust, but recovery trajectories after a recent disease (e.g., influenza, cardiac decompensation, or chronic obstructive pulmonary disease exacerbation) may be one of the best available examples of resilience assessment. ${ }^{14)}$ However, the common cold is a milder disease than influenza, and therefore, the plausibility of recovery from common cold as a physical resilience marker needs to be studied further.

This study has some limitations. First, we retrospectively gathered information about recovery from cold; thus, participant recall errors were possible. However, individuals frequently catch the common cold, and self-awareness of days required to recover from a cold is a part of everyday culture. Adults are estimated to experience 2-5 cold events per year; thus, adults aged $>25$ years will have experienced over 100 episodes of common cold during their lives, making the common cold a familiar part of life. ${ }^{15)}$ In ideal circumstances, a clinical examination would also have been conducted; however, a more reasonable approach is the use of self-diagnosis in clinical research studies of common cold because of the familiarity of the subjects with common cold and the lack of a biological definition for common cold. ${ }^{15)}$ Therefore, data on the subjective days required for recovery from a cold are expected to be precise. Second, this study gathered information about colds using a self-report questionnaire; therefore, the answers regarding experiences with common cold might have referred to an illness other than it. ${ }^{16)}$ However, the self-report format is often used in research because people are generally familiar with colds. ${ }^{7}$ Furthermore, the Food and Drug Administration encourages the use of patient-reported surveys that come directly from the patient because they are not biased by interpretations of physicians or others. ${ }^{17)}$ Third, the perceived time required to recover from common cold may be influenced by subjective health and mood. For instance, respondents with lower EQ-VAS scores recalled more days to recov- er from the cold, which could be a recall bias. However, the EQVAS score was adjusted in these multivariate analyses and the possibility of recall bias was low. We also analyzed its association with the BRS score while considering the EQ-VAS score for subjective health in the ANCOVA. However, the ANCOVA did not include mood or depression, which may have led to recall bias. Fourth, while the BRS has been validated for Korean college students, ${ }^{10)}$ it has not yet been validated for Korean older adults. Fifth, although we showed an association between days required for recovery from a cold and the psychological resilience scale score, quality of life score, and physical activity level, these relationships may not be enough evidence to represent physical resilience.

Almost all older adults experience common colds; thus, this common stressor may be useful for evaluating physical resilience. Moreover, patient-reported surveys of common colds are reliable. Therefore, our finding of the association between the average number of days required for recovery from common cold and psychological resilience supports its potential as a marker of physical resilience.

In conclusion, the average number of days required for recovery from common cold was strongly associated with psychological resilience. The number of days required to recover from common cold may be an indirect marker for physical resilience, at least with respect to immunity, as it may represent the recovery capacity after a stressor such as a viral infection. Although the recovery days were not related to the frailty index or number of falls in this cross-sectional study, a longitudinal study is needed to measure health outcomes immediately before and after encounter with a stressor.

\section{ACKNOWLEDGMENTS}

\section{CONFLICT OF INTEREST}

The researchers claim no conflicts of interest.

\section{FUNDING}

The Ministry of Health and Welfare of the Republic of Korea financed this research (No. HI15C3153). The Korean Health Industry Development Institute (KHIDI) provided funding for this study under the Korea Health Technology R\&D Project.

\section{AUTHOR CONTRIBUTIONS}

Conceptualization, CWW, SK; Data curation, YKK, SK; Funding acquisition, CWW; Investigation, CWW, SK; Methodology, CWW, SK, YKK; Writing-original draft, YKK, SK; Writing-review \& editing, YKK, SK, BSK, MJK, JSY, EJJ, HNL, CWW. 


\section{REFERENCES}

1. Won CW. Diagnosis and management of frailty in primary health care. Korean J Fam Med 2020;41:207-13.

2. Smith BW, Dalen J, Wiggins K, Tooley E, Christopher P, Bernard J. The brief resilience scale: assessing the ability to bounce back. Int J Behav Med 2008; 15:194-200.

3. Hale M, Shah S, Clegg A. Frailty, inequality and resilience. Clin Med (Lond) 2019;19:219-23.

4. Whitson HE, Duan-Porter W, Schmader KE, Morey MC, Cohen HJ, Colon-Emeric CS. Physical resilience in older adults: systematic review and development of an emerging construct. J Gerontol A Biol Sci Med Sci 2016;71:489-95.

5. Whitson HE, Cohen HJ, Schmader KE, Morey MC, Kuchel G, Colon-Emeric CS. Physical resilience: not simply the opposite of frailty. J Am Geriatr Soc 2018;66:1459-61.

6. Smith BW, Dalen J, Wiggins K, Tooley E, Christopher P, Bernard J. The brief resilience scale: assessing the ability to bounce back. Int J Behav Med 2008; 15:194-200.

7. Cohen S, Janicki-Deverts D, Doyle WJ. Self-rated health in healthy adults and susceptibility to the common cold. Psychosom Med 2015;77:959-68.

8. Won CW, Lee S, Kim J, Chon D, Kim S, Kim CO, et al. Korean frailty and aging cohort study (KFACS): cohort profile. BMJ Open 2020;10:e035573.

9. Ouchi E, Niu K, Kobayashi Y, Guan L, Momma H, Guo H, et al. Frequent alcohol drinking is associated with lower prevalence of self-reported common cold: a retrospective study. BMC Public Health 2012;12:987.
10. Choi N. Further validation of the Brief Resilience Scale from a Korean college sample. J Asia Pac Couns 2019;9:39-56.

11. Fried LP, Tangen CM, Walston J, Newman AB, Hirsch C, Gottdiener J, et al. Frailty in older adults: evidence for a phenotype. J Gerontol A Biol Sci Med Sci 2001;56:M146-56.

12. Won CW, Lee Y, Lee S, Kim M. Development of Korean Frailty Index for Primary Care (KFI-PC) and its criterion validity. Ann Geriatr Med Res 2020;24:125-38.

13. Duan-Porter W, Cohen HJ, Demark-Wahnefried W, Sloane R, Pendergast JF, Snyder DC, et al. Physical resilience of older cancer survivors: an emerging concept. J Geriatr Oncol 2016;7:4718.

14. Gijzel S, Whitson HE, van de Leemput IA, Scheffer M, van Asselt D, Rector JL, et al. Resilience in clinical care: getting a grip on the recovery potential of older adults. J Am Geriatr Soc 2019; 67:2650-7.

15. Eccles R. Is the common cold a clinical entity or a cultural concept? Rhinology 2013;51:3-8.

16. Bensenor IM, Cook NR, Lee IM, Chown MJ, Hennekens CH, Buring JE, et al. Active and passive smoking and risk of colds in women. Ann Epidemiol 2001;11:225-31.

17. U.S. Department of Health and Human Services FDA Center for Drug Evaluation and Research; U.S. Department of Health and Human Services FDA Center for Biologics Evaluation and Research; U.S. Department of Health and Human Services FDA Center for Devices and Radiological Health. Guidance for industry: patient-reported outcome measures: use in medical product development to support labeling claims: draft guidance. Health Qual Life Outcomes 2006;4:79. 